\title{
El futuro del Derecho del Trabajo
}

\section{The future of Labour Law}

\section{Sergio Quiñones ${ }^{(*)}$}

Pontificia Universidad Católica del Perú

Resumen: La premisa del presente artículo es que el futuro del trabajo definirá el futuro del Derecho del Trabajo, en tanto este tiene por objeto esencial la regulación de aquél. Desde esa perspectiva, se analizan las mega tendencias identificadas por la Organización Internacional del Trabajo que seguirán impactando el mundo del trabajo: la globalización, el incremento de la tasa de dependencia, la innovación tecnológica y, sobre todo, el cambio climático. Asimismo, el artículo aborda los principales problemas laborales que aún afrontan los países latinoamericanos, así como los retos que se le presentan en el futuro próximo al Perú a fin de cerrar la brecha entre la cantidad de empleo que se genera en el país y la calidad de dicho empleo.

Palabras Clave: Trabajo - Futuro - Organización Internacional del Trabajo - Mega-Tendencia - Empleo - Calidad

\begin{abstract}
The premise of this paper is that the future of work will define the future of Labour Law, since work is indeed the main object of regulation of such legal discipline. From this perspective, we analyze the mega trends identified by the International Labour Organization that will continue to impact the world of work: globalization, the increase in the dependency rate, technological innovation and, above all, climate change. Likewise, the article addresses the main labor problems still facing Latin American countries, as well as the challenges that are presented in the near future to Peru in order to close the gap between the amount of employment generated in the country and the quality of said employment.
\end{abstract}

Keywords: Work - Future - International Labour Organization - Mega-Trend - Employment - Quality

Sumario: 1. Introducción_2. El futuro del trabajo y la justicia social_3. El panorama en América Latina_4. La situación en el Perú_5. Conclusiones

(*) Nota del Editor: Este artículo fue recibido el 10 de octubre de 2017 y su publicación fue aprobada el 15 de noviembre de 2017.

$\left(^{* *}\right)$ Magíster en Trabajo y Política Social por la Universidad Autónoma de Barcelona. Profesor de Derecho del Trabajo en la Pontificia Universidad Católica del Perú. Abogado Consejero en Miranda \& Amado y Consultor Externo de la OIT. Miembro Extraordinario de la Asociación IUS ET VERITAS. Correo electrónico: quinones.sa@pucp.edu.pe 


\section{Introducción}

El objeto del presente artículo es reflexionar acerca de los retos y de las oportunidades que, desde nuestra perspectiva, se le pueden presentar en el futuro cercano al Derecho del Trabajo, tomando como punto de referencia el cumplimiento del primer centenario de creación de la Organización Internacional del Trabajo (En adelante, “OIT”) el próximo 2019.

En efecto, a inicios del siglo XX el Derecho del Trabajo no solo atravesó un significativo proceso de constitucionalización, sino que alcanzó su internacionalización a través de la creación de la OIT en el Tratado de Paz de Versalles de 1919, que puso fin a la Primera Guerra Mundial. De allí, pues, la relevancia que tiene para nosotros estas fechas como parámetros temporales de evaluación de lo avanzado y de lo que resta por avanzar en lo que concierne a esta disciplina jurídica.

Más aún si tenemos en cuenta que a inicios del siglo pasado existía una alta valoración de los intereses tutelados por el Derecho Laboral, y que el trabajo era considerado determinante para la garantía de paz y justicia social que se perseguía luego de la Primera Guerra Mundial. Baste para ello recordar que en el Preámbulo de la Constitución de la OIT se señaló que la paz universal y permanente sólo podía basarse en la justicia social y que existían condiciones de trabajo que entrañaban tal grado de injusticia para gran número de seres humanos, que el descontento causado constituía una amenaza para la paz y armonía universal.

En contraposición, las últimas décadas del siglo $X X$ nos mostraron cómo la valoración económica y social del trabajo se enfrentó a la grave crisis económica de las décadas de los setenta y ochenta, lo que originó que la flexibilidad laboral se consolide como la política laboral predominante en la mayoría de países por las próximas dos siguientes décadas.

Cabe aquí citar las reflexiones del sociólogo alemán Ulrich Beck acerca de los efectos que la posmodernidad o sociedad del riesgo generaron en el mundo del trabajo:

"las flexibilizaciones de la jornada laboral y del lugar de trabajo difuminan los límites entre el trabajo y el no trabajo (...) con ello se eliminan mediante la modernización las premisas jurídicas y sociales anteriores del sistema de ocupación: el desempleo masivo es 'integrado' en el sistema de ocupación mediante nuevas formas de 'infra ocupación plural', con todos los riesgos y oportunidades que esto conlleva" (1998, 20).

Bajo esta línea de razonamiento, Beck critica fuertemente el mito según el cual la sociedad industrial desarrollada durante el siglo $X X$, con su esquema de trabajo y vida, sectores productivos y categorías de crecimiento económico, constituya un punto culminante del desarrollo, o que sea una sociedad completamente moderna (Beck 1998, 17); por el contrario, lo que el autor alemán sostiene es que "el sistema de coordenadas en que descansan la vida y el pensamiento en la modernidad industrial (los ejes de familia y trabajo, fe en la ciencia y en el progreso) empieza a oscilar, y surge un nuevo juego de oportunidades y riesgos, los contornos de la sociedad del riesgo" (Beck 1998, 21).

De este modo, a través del estudio de conceptos como la desestandarización del trabajo productivo y de fenómenos como el tránsito de un sistema de pleno empleo a uno de subempleo flexible y plural, Beck nos remitía ya a una realidad palpable el día de hoy, sobre todo en mercados laborales como el peruano, en la que predominan altos niveles de sub ocupación, informalidad y desprotección social de grandes capas de trabajadores.

Así es como hemos llegado a los albores del siglo XXI, cuando, tras avances y retrocesos de la ola flexibilizadora, podemos tener relativa certeza respecto a que el concepto de trabajo decente ocupa el día de hoy un eje central en torno al cual giran las prioridades de la agenda laboral internacional de la mayoría de Estados miembros de la OIT, incluido ciertamente el Perú.

Dicho esto, en las próximas páginas nos proponemos analizar la agenda que el futuro cercano le depara al Derecho del Trabajo, tanto desde la perspectiva de la propia OIT como desde la mirada regional y nacional. 
Sergio Quiñones

\section{El futuro del trabajo y la justicia social}

Como fue previamente comentado, el Tratado de Paz de Versalles de 1919 dispuso en su capítulo XIII la creación de la OIT bajo la premisa de que la paz universal y permanente solo podía basarse en la justicia social.

Con ese mandato, la OIT ha erigido un sistema de normas internacionales en todas las materias relacionadas con el trabajo orientadas a la consecución de dicha justicia social, de modo tal que en sus casi cien años de existencia se han internacionalizado las principales normas socio laborales en temas como jornada de trabajo, protección de la maternidad, seguridad y salud ocupacional, abolición del trabajo forzoso o prevención del trabajo infantil, entre otros instrumentos destinados a promover mejores condiciones laborales a nivel global.

Uno de los hitos recientes y, en nuestra opinión, más importantes en este devenir ha sido la consolidación y extensión del concepto de trabajo decente mediante la Declaración de la Organización Internacional del Trabajo sobre la justicia social para una globalización equitativa, adoptada el 10 de junio de 2008.

Ciertamente, a partir de la Declaración en cuestión, los gobiernos, las organizaciones de empleadores y las de trabajadores reconocen que el trabajo decente constituye un elemento central de las políticas económicas y sociales, y que debe basarse en cuatro objetivos estratégicos: (i) generar empleos en un entorno institucional y económico sostenible; (ii) respetar, promover y aplicar los principios y derechos fundamentales en el trabajo; (iii) adoptar y ampliar medidas de seguridad social y protección de los trabajadores, que sean sostenibles y estén adaptadas a las circunstancias nacionales; y, (iv) fortalecer el diálogo social y el tripartismo.

Precisamente, como señala la propia OIT:

"la categoría 'trabajo decente' obliga a considerar los principales aspectos del trabajo y de las relaciones laborales. Es imposible separar un aspecto, como la generación de empleo o la defensa de los derechos laborales, de los otros aspectos. El conjunto de características que implica esta noción abarca simultáneamente el cumplimiento y aplicación de las normas, la promoción del empleo, la extensión de la protección social y el impulso del diálogo social" (OIT 2003, 9).

Bajo esta lógica de conseguir que la justicia social sea el objetivo primordial para todos los mandantes de la organización, otro hito que consideramos igual de relevante, pero bastante más reciente, ha sido el lanzamiento de la Iniciativa del Centenario relativa al Futuro del Trabajo.

Efectivamente, en la Memoria del Director General elaborada con motivo de la 104 reunión de la Conferencia Internacional del Trabajo realizada el año 2015 , se señala que "por definición, la iniciativa debe ser una contribución a la causa de la justicia social. Su importancia particular -y tal vez el interés que ha suscitado- radica en que ha surgido en un contexto de gran incertidumbre e inseguridad, y del temor de que la evolución del mundo del trabajo lo aleje del objetivo de la justicia social, en lugar de acercarlo" (OIT, 2015, 2).

A efectos de poner en práctica esta iniciativa y alcanzar las metas propuestas, el Director General de la OIT ha planteado la ejecución de tres fases principales. La primera busca generar un debate y reflexión de alcance global en torno al futuro del trabajo, y para ello se ha invitado a participar a los mandantes tripartitos, a otras organizaciones internacionales, instituciones de investigación, universidades y sociedad civil en general.

La segunda fase consiste en la creación de la Comisión Mundial sobre el Futuro del Trabajo, cuya finalidad es encargarse de evaluar y sistematizar los aportes y reflexiones recibidos durante la primera fase, encargo que quedará plasmado en un informe de alto nivel que se presentará en la 108 reunión de la Conferencia Internacional del Trabajo, el 2019. 


\section{El futuro del Derecho del Trabajo The future of Labour Law}

Precisamente, en el año del centenario de creación de la OIT se llevaría a cabo la tercera fase de la iniciativa, en la cual se establece lo siguiente:

"se alentaría a todos los Estados Miembros a que
organicen eventos relacionados con el centenario de la
OIT durante el primer semestre del año. Los mandantes
tripartitos nacionales sin duda querrán incluir una
dimensión conmemorativa o histórica en esos eventos en
función de la interacción que hayan mantenido con la OIT a
lo largo de los años. Pero también es importante que todos
y cada uno de ellos dejen lugar para los temas derivados
de la iniciativa relativa al futuro del trabajo" (OIT 2015, 2).

Tal como se mencionó, la creación de la Comisión Mundial sobre el Futuro del Trabajo marcó el inicio de la segunda etapa de esta iniciativa de la OIT, y su función principal ha sido realizar un examen profundo sobre el futuro del trabajo, teniendo siempre como horizonte el mandato de la organización en materia de justicia social para el siglo XXI.

Así, el 4 de diciembre de 2017 se publicó en la página web institucional de la OIT el Inception Report for the Global Commission on the Future of Work, donde la citada Comisión ofrece una panorámica preliminar de las más importantes mega tendencias globales que están transformando el mundo del trabajo, así como de las problemáticas más relevantes que lo afectan.

En suma, la Comisión Mundial sobre el Futuro del Trabajo destaca que "technology, demography, globalization and climate change are key mega drivers of change in the world of work. Looking ahead, we need to understand how these megatrends will affect work and society in the future and how best to harness these trends to generate opportunities (OIT 2017a, 8)(1)".

En lo que concierne particularmente a la globalización, el informe preliminar de la Comisión señala que aquella sea tal vez la característica más relevante de la economía mundial en las últimas décadas, comprendiendo bajo dicho fenómeno tanto a la internacionalización de la producción de bienes y servicios, propiamente dicha, como a la de las finanzas, el comercio y la migración de personas.

En esa línea, la Comisión destaca que la globalización ha generado cambios importantes en los patrones de producción global, sobre todo en el sector terciario o de servicios, lo que ha incidido directamente en la fragmentación -tercerización- de la producción en tareas y actividades y la consecuente expansión de las cadenas mundiales de suministro. Ciertamente, estos cambios originados en la globalización se han visto facilitados por la reducción de los costos de comercio y transporte, así como por la innovación tecnológica (OIT 2017a).

Por su lado, la relevancia de la tecnología, como una de las principales mega tendencias, está ciertamente vinculada al enorme impacto que el cambio tecnológico está generando, no solo en la expansión y evolución del conocimiento, sino directamente en el mercado laboral.

El informe de la Comisión alude a la denominada cuarta revolución industrial, la big data, la impresión tridimensional, la inteligencia artificial y la robótica, por nombrar solo algunos casos que están teniendo un impacto transformador en la naturaleza del trabajo actual. Se señala, por ejemplo, que desde el año 2010 el número de robots industriales ha aumentado en un $9 \%$ en promedio anual, llegando a unos 1,6 millones de unidades al final de 2015. Aunque, es preciso resaltar, el $80 \%$ de la producción y utilización de robots para la industria está concentrado en los países desarrollados (OIT 2017a).

Respecto a la demografía, el informe de la Comisión llama nuestra atención sobre el incremento sostenido de la tasa de dependencia (proporción de personas de 65 años o más frente a las de 15 a 64 años).

(1) Traducción libre del autor: "tecnología, demografía, globalización, y cambio climático constituyen las mega-tendencias globales del cambio en el mundo del trabajo. De cara al futuro, debemos comprender cómo estas mega-tendencias afectarán el trabajo y la sociedad en el futuro y cómo podemos aprovecharlas de la mejor manera para generar oportunidades." 


\section{Sergio Quiñones}

Así, se indica que, mientras en 1950 había alrededor de 8 personas mayores de 65 años por cada 100 personas en edad de trabajar, en 2015 dicha cifra aumentó a más de 12 y se proyecta que llegará a 18 en 2030.

De este modo, la transición a una sociedad y fuerza de trabajo mayores y la importancia cambiante de las cohortes de diferentes edades, pueden generar cambios significativos en la propia percepción del trabajo y, sobre todo, demandar de los gobiernos una reevaluación de los sistemas de seguridad social basados en el principio de solidaridad intergeneracional. El otro lado de la moneda lo constituye el hecho de que, una esperanza de vida más larga de la población podría generar oportunidades nuevas de empleo en sectores directamente involucrados con el cuidado de la salud y la atención a la dependencia (OIT 2017a).

Finalmente, la cuarta y última mega tendencia de cara al futuro del mundo del trabajo lo constituye, qué duda cabe, el cambio climático. Sobre el particular, el informe de la Comisión nos alerta acerca de cómo, en el tiempo reciente, la población global ha utilizado más recursos y generado más desechos de los que el planeta y nosotros mismos podemos regenerar y absorber.

Ello ha tenido consecuencias claramente devastadoras en el medio ambiente, como la contaminación atmosférica, del agua y la pérdida de biodiversidad; pero además debe tenerse en consideración que los cambios en la temperatura, en los niveles de agua y los desastres climáticos extremos, como inundaciones y sequías, han agravado también los problemas que enfrenta el sector agrícola, y, con ello, la sostenibilidad de los sistemas alimentarios y la seguridad alimentaria mundial (OIT 2017a).

Atendiendo a lo antes dicho, la Comisión sostiene que existe un fuerte consenso de que el futuro del trabajo y la sociedad deben basarse en un desarrollo ambientalmente sostenible, tanto en el consumo como en la producción. Para ello, los trabajos de energía renovable pueden proporcionar el reemplazo necesario en la transición hacia una economía más verde; mientras que los empleos existentes deberán adaptarse a los requisitos de una economía más ecológica. La idea, a fin de cuentas, es que se produzcan medios de transporte más eficientes en combustible, que el sector agrícola sea más resiliente al clima y que los servicios adopten técnicas más eficientes en el uso de energía; tales medidas reflejan las transformaciones necesarias en el mundo del trabajo para alcanzar los objetivos del cambio climático (OIT 2017a).

Precisamente, sobre la premisa de la existencia de un consenso sólido y global respecto a un futuro del trabajo ambientalmente sostenible, la OIT ha venido desarrollando en paralelo la Iniciativa del Centenario Verde, cuyo objetivo es comprender mejor los desafíos y oportunidades que esta transición conlleva y formular recomendaciones efectivas de políticas basadas en evidencia.

En el marco de dicha iniciativa, los resultados de las investigaciones determinaron que era posible incrementar la tasa global de empleo de 0,5 a $2 \%$ hacia el 2030 , particularmente en los sectores de la agricultura, la silvicultura, la energía, el reciclaje, la construcción y el transporte (OIT 2017b). Más concretamente, la OIT se refiere al caso de la Unión Europea, en donde se data lo siguiente:

"las empresas de la 'ecoindustria' empleaban a más de 4,2 millones de personas en 2013, un nivel muy superior al de las industrias del automóvil, los textiles o la industria química. Las empresas del sector de los productos y servicios ecológicos generaron beneficios superiores a 700000 millones de euros ese mismo año. La Unión Europea considera que hay un crecimiento de los empleos verdes, y que integrando más eficazmente las preocupaciones relativas al medio ambiente y el cambio climático en las políticas de energía y formación se podrían obtener aún más beneficios en términos de creación de empleos" (OIT 2017b, 8).

Respecto a los sectores específicos de actividad económica, el estudio desarrollado por la Iniciativa del Centenario Verde destaca 
el caso del sector del transporte debido a que, cambios como el abandono de los automóviles particulares a favor del transporte público, estarían teniendo efectos notables en la infraestructura y el incremento del número de empleos.

Igualmente,

"la expansión de la producción agrícola orgánica también ha demostrado su potencial para impulsar el empleo y los ingresos. En una encuesta de 2014 de la Federación Internacional de los Movimientos de Agricultura (IFOAM) se determinó que había un total de 2,3 millones de productores de cultivos agrícolas orgánicos en todo el mundo - tres cuartas partes de los cuales se encontraban en los países en desarrollo y emergentes" (OIT 2017b, 9).

Por último, el turismo es otro sector de actividad económica en donde se pueden apreciar interesantes estrategias de adaptación que han contribuido a generar un sostenido crecimiento del turismo no tradicional y en pequeña escala como alternativa al turismo de masa; destacando como ejemplo de ello el aumento del ecoturismo, el turismo de aventura, y el cultural, como oportunidades de creación de nuevos empleos (OIT 2017b).

En síntesis, podemos culminar el presente apartado sosteniendo que, a puertas de su primer centenario, la OIT sigue impulsando institucionalmente la consecución de la justicia social a través del trabajo decente, y que en ese objetivo deben tomarse en consideración las principales tendencias globales que hoy impactan, y seguirán impactando, el mundo del trabajo, como es la globalización de la economía, el incremento de la tasa de dependencia, la innovación tecnológica y, sobre todo, el cambio climático; siendo este último fenómeno el que más debería preocupar a los estados miembro y a los diversos actores sociales, de cara a conseguir que el futuro del trabajo sea ambientalmente sostenible.

\section{El panorama en América Latina}

El Latinobarómetro 2017 es un estudio de opinión pública que recoge los resultados sistematizados de haber aplicado, entre junio y agosto del año indicado, un total de 20,200 entrevistas en 18 países con muestras representativas del $100 \%$ de la población nacional de cada país.
Dicho estudio resulta particularmente relevante para el presente artículo no solo porque se trata de una encuesta que viene realizándose de manera continuada desde el año 1995 sino también debido a la diversidad temática característica de sus entrevistas, comprendiendo en este caso temas como percepción democrática, cambio climático y perspectiva económica, que es donde específicamente nos centraremos en los siguientes párrafos.

Un primer indicador que nos interesa destacar es el de la percepción de perder el empleo, que permite observar cuál es el horizonte económico de la población económicamente activa (ver Gráfico 1). De este modo, "ante la pregunta si está preocupado de perder el empleo en los próximos doce meses, un $76 \%$ contestaba que sí en 1995 , mientras sólo un $41 \%$ lo hace en 2017 . Este indicador ha disminuido 35 puntos porcentuales en 22 años, mostrando un mercado del trabajo más estable" (Latinobarómetro 2017, 51).

Según el estudio en cuestión, existiría, además, una relación directa y positiva entre la percepción de estabilidad en el empleo y una mejora en los ingresos que se traduce en la capacidad de ahorro como fenómeno social, de tal suerte que "el empleo más allá de los doce meses hace posible tener un horizonte económico que incluye el concepto de ahorro" (Latinobarómetro 2017, 51).

No obstante, pese a la sustantiva mejora en este indicador de estabilidad en el empleo, el $23 \%$ de latinoamericanos encuestados afirmó que el principal problema de su país era de naturaleza económica (lo que incluye los bajos salarios, la falta de empleo, la pobreza); y un $11 \%$ adicional señaló que lo era la economía o las finanzas en general (ver Gráfico 2); es decir, que "en total, $34 \%$, uno de cada tres latinoamericanos, afirma que el principal problema que enfrenta su país es de tipo económico" (Latinobarómetro 2017, 58). 
Sergio Quiñones

\section{Gráfico 1}

IMAGEN DE PROGRESO EN EL PAÍS

TOTAL AMÉRICA LATINA 2017- TOTALES POR PAIS 2017

¿ ¿iiria Ud. que este pais... Está progresando, Está estancado, Está en retroceso. Aqui solo "Está progresando".

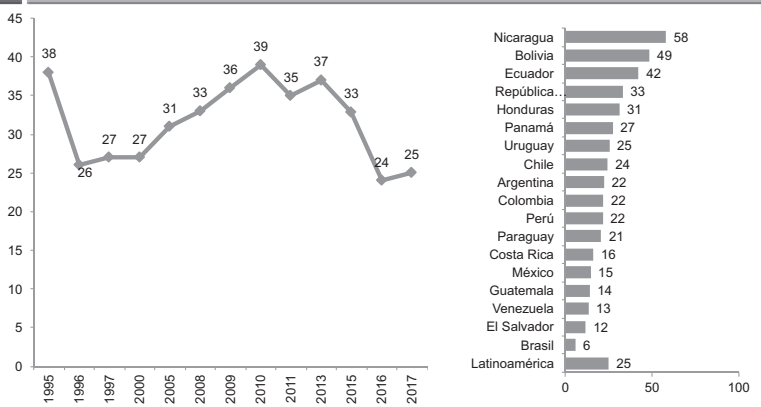

\section{Gráfico 2}

\section{PROBLEMA MÁS IMPORTANTE DEL PAÍS}

\section{TOTAL AMÉRICA LATINA 2017}

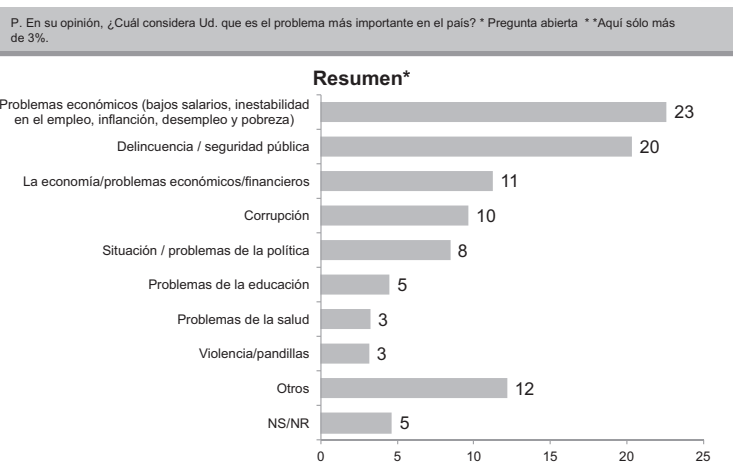

Una posible explicación para la aparente contradicción que los dos indicadores antes comentados parecen evidenciar, la podemos encontrar en un reciente análisis que el profesor Carlos Parodi realizó respecto al índice de ingreso por habitante en la región; esto es, de cuánto, en promedio, recibe un ciudadano en un país a lo largo de un año (Parodi 2017).
Según el citado autor, y sobre la base de datos de CEPAL ${ }^{(2)}$ para el año 2015, Chile es el país de América Latina con mayor ingreso por habitante (14,547 dólares), seguido de lejos por Perú con 5,935 dólares, y teniendo en la cola a Bolivia y Haití con 2,390 dólares y 738 dólares, respectivamente. Como se evidencia, "América Latina no solo es heterogénea (usando el indicador descrito), sino que además es la región más desigual del mundo" (Parodi 2017).

Profundizando en ello, Parodi nos recuerda que la metodología más usada para medir la desigualdad es el coeficiente de Gini, siendo 0 indicador de mayor igualdad y 1 de mayor desigualdad. Así, América Latina tiene un coeficiente de Gini promedio de 0.50 para el año 2015, bastante por encima del de Noruega (0.25), Estados Unidos (0.41) e, incluso, del de China (0.42).

En palabras del autor, el indicador antes comentado

"significa, que, dentro de América Latina, existen muchos ciudadanos concentrados en los dos extremos (o en uno de ellos), lo que hace que el promedio sea menos representativo que en otros países. Los países con menor Gini, son aquellos que invierten más y mejor en educación y salud y en general, en temas directamente relacionados con el bienestar de los habitantes. Son sociedades menos conflictivas, en las que se puede llegar de manera más fácil a consensos. Por eso es que es más simple crecer. Un hecho empírico es que los países con mayor desigualdad de ingresos crecen menos que los más igualitarios. Más aún, a mayor desigualdad de ingresos, menor impacto del crecimiento sobre la reducción de la pobreza monetaria" (Parodi 2017).

(2) Véase Comisión Económica para América Latina y el Caribe (https://www.cepal.org/es). 
Es decir, podríamos formular cómo hipótesis que, si bien existe en la región un nivel adecuado de empleo y de ocupación con cierta estabilidad en el tiempo, dichos trabajos no necesariamente estarían generando los ingresos (salarios) suficientes en favor de la población como para que los problemas económicos dejen de ser su principal preocupación cotidiana.

Precisamente, el Banco Interamericano de Desarrollo (En adelante, "BID") ha publicado recientemente el Índice de Mejores Trabajos, que constituye una herramienta para el análisis comparativo de los mercados laborales en América Latina.

Lo interesante y novedoso de este índice es que evalúa la situación del empleo en los países de América Latina a través de dos dimensiones: (i) cantidad; y, (ii) calidad. Cada una de estas dimensiones, a su vez, está conformada por dos indicadores, según se detalla a continuación:

De este modo, con el Índice de Mejores Trabajos, el BID busca superar la tradicional forma de medir las condiciones de empleo en una economía, que se ha basado normalmente en la tasa de desempleo (cuántas personas de las que participan activamente en el mercado laboral no consiguen trabajo), y con ello introducir dimensiones de calidad mucho más significativas de cara a lo que veníamos analizando párrafos atrás: la desigualdad de ingresos y su impacto en la pobreza de los países de América Latina.
En este punto, consideramos pertinente detenernos en la definición que el BID ha propuesto para el indicador de salario suficiente, habida cuenta que un empleo con un salario alto será considerado como uno de mayor calidad, mientras que uno que pague sueldo muy bajo será, lógicamente, un trabajo de poquísima calidad.

A tal efecto, para definir un salario suficiente el BID ha considerado dos premisas: (i) una familia promedio de 4 miembros, en la que 1,5 están empleados en una jornada semanal de 48 horas; $y$, (ii) un estándar de vida objetivo equivalente a US\$ 5 por persona por día (Paridad de Poder Adquisitivo [En adelante, "PPA"] 2011), cantidad adoptada como el umbral de la pobreza por el Banco Mundial para la región de América Latina y el Caribe.

Así, por un lado, se considera 5 dólares por día para cada miembro de una familia de 4 , dando como resultado un gasto anual familiar equivalente a 7,300 dólares; por otro lado, tenemos 1,5 trabajadores que laborarán en un año de 52 semanas un total de 3,744 horas. Dividiendo los 7,300 dólares entre 3,744, tenemos como resultado 1.95 dólares por hora. En suma, "si la tasa salarial asociada con este trabajo es igual o superior a 1.95 dólares

\section{Cuadro 1}

\begin{tabular}{|c|c|c|c|}
\hline \multicolumn{2}{|l|}{ Dimensión Cantidad } & \multicolumn{2}{|l|}{ Dimensión Calidad } \\
\hline Participación laboral & Ocupación & Formalidad & Salario suficiente \\
\hline $\begin{array}{l}\text { La tasa de participación laboral se } \\
\text { define como el número de personas } \\
\text { en la población en edad de trabajar } \\
\text { (entre } 15 \text { y } 64 \text { años) que participan } \\
\text { activamente en la fuerza de trabajo } \\
\text { (ya sea en un puesto de trabajo, o } \\
\text { desempleado y en busca de empleo) } \\
\text { dividido por la población total en edad } \\
\text { de trabajar. }\end{array}$ & $\begin{array}{l}\text { Este indicador representa la medida } \\
\text { en que la economía de un país está } \\
\text { generando empleo con éxito. Así, la } \\
\text { tasa de ocupación se define como el } \\
\text { número de personas ocupadas divi- } \\
\text { dido por el número total de personas } \\
\text { en la población en edad de trabajar. }\end{array}$ & $\begin{array}{l}\text { Este indicador mide la capacidad que } \\
\text { tiene una economía de proporcionar } \\
\text { empleos formales. Así, la tasa de for- } \\
\text { malidad consiste en dividir el número } \\
\text { de empleos formales entre el tamaño } \\
\text { de la población en edad de trabajar. }\end{array}$ & $\begin{array}{l}\text { Este indicador mide los salarios } \\
\text { asociados a los trabajos de cada } \\
\text { país. La tasa de trabajos con ingreso } \\
\text { suficiente para superar la pobreza } \\
\text { se consigue dividiendo el número de } \\
\text { puestos de trabajo con salarios dig- } \\
\text { nos entre el tamaño de la población } \\
\text { en edad de trabajar. }\end{array}$ \\
\hline
\end{tabular}

Elaboración propia.

Fuente: Banco Interamericano de Desarrollo https://mejorestrabajos.iadb.org/es/dimensiones 


\section{Sergio Quiñones}

equivalente por hora, entonces diremos que el trabajador está en un puesto de trabajo que paga un salario suficiente" (BID 2017).

Volviendo a los resultados publicados en el Índice de Mejores Trabajos, debemos tomar en consideración que el índice por país es la media ponderada de los 4 indicadores detallados en el Cuadro 1 precedente, y que sus puntuaciones van de 0 a 100 , de tal suerte que un puntaje de 100 supondría que en un país determinado todas las personas que participan en la fuerza laboral están empleadas formalmente y perciben un salario suficiente.

Dicho esto, el índice promedio ponderado de los 4 indicadores para América Latina es de 57 puntos. Sin embargo, lo revelador de estos resultados está en la disparidad que se observa entre las dimensiones de cantidad y calidad en el empleo. Así, mientras que el promedio de la región en materia de participación se eleva a 77 puntos, el promedio de ocupación llega a los 72 puntos; es decir, en lo que respecta a las dimensiones de cantidad, América Latina está, aproximadamente, 22 puntos por encima del índice ponderado.

Muy distinta es la situación al evaluar los indicadores de calidad. Efectivamente, el promedio de la región en materia de formalidad baja sustancialmente hasta los 34 puntos, mientras que el promedio de salario suficiente se ubica en 47 puntos; esto es, que las dimensiones de calidad en el empleo en América Latina están, en promedio, 17 puntos por debajo del índice ponderado de los 4 indicadores.

Para concluir este apartado, nos interesa remitirnos nuevamente a la ya mencionada Iniciativa del Centenario relativa al Futuro del Trabajo, y es que en el caso específico de América Latina se realizaron durante el 2016 distintas encuestas a los jóvenes latinoamericanos en las que se abordaron los principales ejes temáticos comprendidos en la primera fase de la Iniciativa en cuestión.

El resultado de dichas encuestas ha sido sistematizado y publicado en un informe de la Oficina Regional para América Latina y el Caribe de la OIT cuya finalidad es brindarnos "una primera aproximación a las percepciones y expectativas de los jóvenes latinoamericanos sobre el futuro del trabajo con base en los resultados de estas encuestas (...) y a partir de estos hallazgos se extrapolan algunas conclusiones, que esperemos permitan alimentar y profundizar el debate sobre el futuro del trabajo" (OIT, 2017c: 8).

A modo de resumen, las principales conclusiones que el referido informe recoge, y que se vinculan a las materias abordadas en el presente artículo, son las siguientes: (i) los jóvenes latinoamericanos enfrentan obstáculos para su plena inclusión en la sociedad y las brechas en el mercado laboral son más significativas que las que enfrentan los adultos en la región; (ii) las mujeres jóvenes, los jóvenes $n i-n i^{(3)}$ y los que trabajan en el sector informal y áreas rurales constituyen los principales grupos vulnerables; (iii) existe una marcada preferencia por trabajar en sus propias empresas en el futuro (69\%), un número igual de elevado (59\%) aspiraría a tener horarios laborales flexibles y otro porcentaje similar (40\%) a trabajar desde su casa (OIT 2017c, 34-9).

En suma, la OIT propone que, para estar a la altura de las expectativas de las y los jóvenes de la región, los países de América Latina requieren desplegar importantes esfuerzos para

\footnotetext{
"aumentar la calidad y pertinencia de la educación y de la formación profesional, con vistas a incrementar la empleabilidad de los jóvenes (...) también es esencial fortalecer los marcos institucionales y de políticas para la juventud que consideren los análisis de prospectiva (demográfica, tecnológica y productiva) y los pongan al servicio de los jóvenes para que puedan tomar decisiones informadas sobre sus
}

(3) Jóvenes que no están empleados, no cursan estudios ni reciben capacitación. 
trayectorias laborales, considerando la evolución de la economía, las empresas y la sociedad" (OIT 2017c, 41).

\section{La situación en el Perú}

En el apartado anterior nos remitimos al Índice de Mejores Trabajos (BID 2017) a fin de evaluar la situación general del mercado laboral latinoamericano desde la perspectiva de los indicadores de cantidad y de calidad. Retomamos ahora los resultados del referido índice a fin de evaluar la situación particular de nuestro país ${ }^{(4)}$.

En el Perú, el índice promedio ponderado de los 4 indicadores (ver Cuadro 1 infra) es de 55 puntos, ubicándose por debajo del promedio de América Latina (57 puntos) y en el puesto 12 de un total de 17 países de la región evaluados.

Al igual que a nivel regional, en el caso peruano se evidencia también una significativa brecha entre los resultados de las dimensiones de cantidad y calidad en el empleo. En efecto, Perú obtiene significativamente más puntos tanto en la tasa de participación (82 frente al promedio regional situado en 77) como en la de ocupación ( 79 frente al promedio regional que es 72); mientras que, en términos de calidad, el puntaje obtenido por nuestro país disminuye sustancialmente en la tasa de formalidad laboral (solo 17 puntos frente al promedio regional de 34) y en el indicador de trabajos con salario suficiente para superar la pobreza (43 frente al promedio regional que se sitúa en 47 ).

En resumen, Perú ocupa la 2 posición en América Latina en la dimensión de cantidad, con 80 puntos, mientras que se ubica en la 12 posición en la dimensión de calidad con solo 30 puntos.

Consideramos que esta importante brecha (50 puntos) entre ambas dimensiones de la evaluación del mercado laboral ratifica la hipótesis formulada previamente, en el sentido de que, así como en la región, también en nuestro país existen tasas importantes de participación y ocupación, pero el problema radica en que dichos empleos no son necesariamente formales ni aseguran a la población un nivel de ingresos suficiente para superar la pobreza.

De hecho, considerando que en Perú el salario mínimo mensual legal asciende en la actualidad a 264 dólares (aproximadamente), el valor hora mínimo de trabajo en el país sería equivalente a 1.37 dólares; esto es, un $30 \%$ inferior a los 1.95 dólares de valor hora que el BID señala como el mínimo indispensable para hablar de un salario suficiente (BID, 2017).

En nuestra opinión, cerrar estas brechas entre la cantidad de empleo que se genera en el país y la calidad de dicho empleo, que es el indicador que de forma más directa repercute en el bienestar de los ciudadanos, constituye uno de los retos más trascendentales que el Estado deberá afrontar en el futuro próximo, abordándolo desde la propia dinámica económica del mercado laboral, pero también desde el Derecho del Trabajo, en tanto conjunto de normas que lo regulan.

Bajo dicha premisa, conviene citar aquí el primer Informe Nacional Voluntario sobre la implementación de la Agenda 2030 para el Desarrollo Sostenible, que ha sido elaborado por el Centro Nacional de Planeamiento Estratégico (En adelante, "CEPLAN"), y presentado el pasado mes de febrero de 2017 para generar un espacio institucionalizado de diálogo y concertación entre todos los actores de la sociedad y del Estado.

La importancia de este informe nacional es doble. En primer lugar, constituye una reafirmación del compromiso del Estado peruano con la Agenda $2030^{(5)}$ y narra cómo ésta se está implementando inicialmente para el logro de sus objetivos. En segundo término, constituye un análisis prospectivo para elaborar una propuesta de imagen de

(4) Para el caso peruano, el Índice de Mejores Trabajos toma como fuente el Sistema de Información de Mercados Laborales y Seguridad Social (SIMS) del propio BID, con datos publicados por el Instituto Nacional de Estadística e Informática (INEI) del último año disponible: 2015. 
Sergio Quiñones

futuro del país al 2030 que guíe la actualización de su Plan Estratégico de Desarrollo Nacional, en armonía con las políticas de Estado de largo plazo y los Objetivos de Desarrollo Sostenible (En adelante, "ODS") (CEPLAN 2017).

En este sentido, la propuesta de imagen de futuro elaborada por CEPLAN se encuentra

"asociada a las cinco esferas de desarrollo sostenible identificadas en el preámbulo de la Agenda 2030 que es base para la construcción de la visión concertada de futuro del Perú al 2030. Para transmitir mejor el sentido de esa propuesta de imagen, se ha identificado una lista de indicadores ilustrativos, directamente relacionadas con cada ODS, a fin de facilitar el diálogo para la concertación de la visión de futuro" (CEPLAN 2017, 20).

En efecto, de acuerdo a la ya mencionada Agenda 2030, los 17 ODS se agrupan en 5 esferas críticas para el desarrollo sostenible: personas, planeta, prosperidad, paz y alianzas. Dentro de la esfera de prosperidad está comprendido el ODS No. 8, consistente en promover el crecimiento económico sostenido, inclusivo y sostenible, el empleo pleno y productivo y el trabajo decente para todos.

Bajo ese mandato, el CEPLAN ha propuesto que, en la esfera de prosperidad, la imagen de futuro del Perú al 2030 sea la de un país en el que "todas las personas gozan de una vida próspera y plena, con empleo digno y en armonía con la naturaleza, considerando reserva de recursos para el bienestar futuro" (CEPLAN 2017, 21); y, para ello, ha establecido 3 indicadores ilustrativos, que servirán para el seguimiento y monitoreo del cumplimiento efectivo de la meta propuesta: (i) tasa de crecimiento anual del PBI real per cápita (\%); (ii) porcentaje de la población ocupada con empleo formal (\%); y, (iii) coeficiente GINI de ingresos (índice).

\section{Conclusiones}

a) El futuro del trabajo definirá el futuro del Derecho del Trabajo, en tanto este tiene por objeto esencial la regulación de aquél.

b) Desde la OIT se reafirma, de cara al cumplimiento de su primer centenario, el compromiso con la justicia social a través del trabajo decente, y se reconoce que las mega tendencias globales que seguirán impactando el mundo del trabajo son la globalización de la economía, el incremento de la tasa de dependencia, la innovación tecnológica y, sobre todo, el cambio climático; siendo el principal reto conseguir que el futuro del trabajo sea ambientalmente sostenible.

c) Los principales problemas que aún afrontan los países latinoamericanos son de naturaleza económica: bajos salarios, falta de empleo, y pobreza en general. El reto en la región radica en reducir la brecha entre la importante creación cuantitativa de empleo y los ingresos insuficientes que dicho empleo genera; y para ello se requiere aumentar la calidad y pertinencia de la educación, la formación profesional, y la empleabilidad de los jóvenes.

d) En el Perú la evidencia también demuestra que la creación de empleo no necesariamente estaría generando los salarios suficientes en favor de la población (estamos en el puesto 12 de 17 países en materia de calidad de empleo). Cerrar esta brecha entre la cantidad de empleo que se genera en el país y la calidad

(5) La Agenda 2030 para el Desarrollo Sostenible de las Naciones Unidas fue adoptada en septiembre de 2015 y dio lugar a 17 Objetivos de Desarrollo Sostenible (ODS) y a 169 metas conexas que representan enormes desafíos para las realidades nacionales de todos los estados miembros, en tanto se propone no dejar a nadie atrás, es decir, continuar con las políticas de erradicación de la pobreza y profundizar el pleno cumplimiento de los derechos humanos. Para mayor información, véase: Naciones Unidad, "La Asamblea General adopta la Agenda 2030 para el Desarrollo Sostenible," http://www.un.org/ sustainabledevelopment/es/2015/09/la-asamblea-general-adopta-la-agenda-2030-para-el-desarrollo-sostenible/ 


\section{El futuro del Derecho del Trabajo}

The future of Labour Law

de dicho empleo constituye uno de los retos más importantes de cara al 2030 , a fin de alcanzar la visión de un Perú en el que todos tengan un trabajo decente $y$, además, en armonía con el medio ambiente.

\section{Referencias bibliográficas}

Beck, Ulrich. 1998. La sociedad del riesgo. Hacia una nueva modernidad. Ediciones Paidós, Barcelona.

Banco Interamericano de Desarrollo. 2017. Índice de Mejores Trabajos. En https:// mejorestrabajos.iadb.org/es. Consultado el 08 de noviembre del 2017.

Centro Nacional de Planeamiento Estratégico. 2017. Informe Nacional Voluntario sobre la implementación de la Agenda 2030 para el Desarrollo Sostenible.

Latinobarómetro. 2017. Latinobarómetro:
Informe 2017. En http://www.latinobarometro.org/lat.jsp. (Consultado el 21 de noviembre del 2017).

Organización Internacional del Trabajo. 2003. Perú: Propuesta de Programa Nacional de Trabajo Decente, 2004-2006. Informe Preliminar. Lima.

2015. La iniciativa del centenario relativa al futuro del trabajo. Memoria del Director General. Ginebra.

2017a. Inception Report for the Global Commission on the Future of Work. Geneva.

2017b. Trabajo y cambio climático: La iniciativa verde. Memoria del Director General. Ginebra.

2017c. El futuro del trabajo que queremos. La voz de los jóvenes y diferentes miradas desde América Latina y el Caribe. Lima: OIT Américas Informes Técnicos 2017/7.

Parodi, Carlos. 2017. Ingreso por habitante y Desigualdad de Ingresos. En http://blogs.gestion.pe/economiaparatodos/2017/06/ ingreso-por-habitante-y-desigualdad-de-ingresos.html (consultado el 21 de junio del 2017). 